
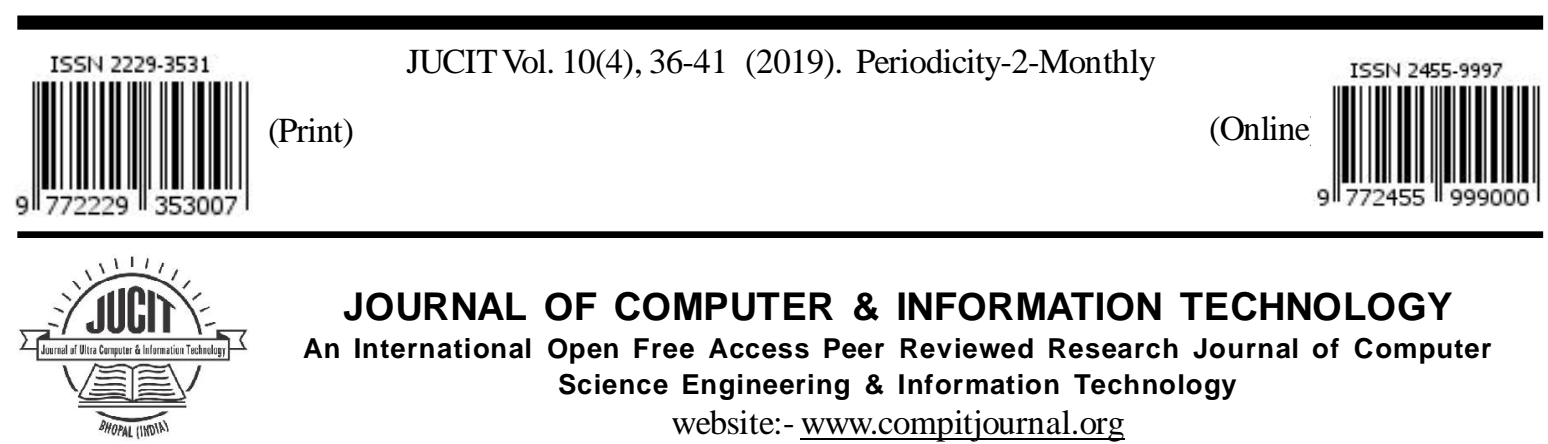

JOURNAL OF COMPUTER \& INFORMATION TECHNOLOGY

An International Open Free Access Peer Reviewed Research Journal of Computer Science Engineering \& Information Technology

Estd. 2010 website:- www.compitjournal.org

\title{
Mitigating Carbon Footprint via Efficient Green Cloud Computing: A Review
}

\author{
SAFWANA HAQUE ${ }^{1}$ and FARHANA HAQUE ${ }^{2 *}$ \\ ${ }^{1}$ Department of Computer Science and Engineering, Brac University Dhaka (Bangladesh) \\ ${ }^{2}$ Department of Computer Science and Engineering, Anwer Khan Modern University Dhaka (Bangladesh) \\ Email address of Corresponding Author: farhanahaque2003@ gmail.com \\ http://dx.doi.org/10.22147/jucit/100401
}

Acceptance Date 26th July, 2019， Online Publication Date 2nd August, 2019

\begin{abstract}
The huge percentage of dependency on computerisation in the present world has led to adverse effects on the global climate and environment beyond the imagination of mankind, awareness of which, interestingly is little. Consumers often wonder what kind of effects computerisation can cause since computers, internet, storage devices, etc. do not emit smoke like vehicles which is known to be the commonest example of greenhouse gas emission. This paper brings forth the effects caused by a recent growing field in the world of computing known as cloud computing in relation to global warming; the various types of researches carried out to overcome and handle the growing issues; making edge computing environmental friendly by reducing energy consumption, carbon gas emission, e-waste disposal and recycling in relation to green computing or green IT, which encompasses of environmental sustainable practices. Green edge computing aims at increasing energy efficiency, reducing environmental hazards, improving life span and working efficiency of devices in cloud data centres and reducing electronic waste disposal and implementation of which is hoped to have positive impacts on the global climate.

Keywords : Carbon Footprint, Cloud computing, Edge Computing, e-Waste Disposal.

\section{Introduction}

With the immense advancement in technology, there has been a rapid development in the sector of information and communication technology. Every sector and field of work and life

have now become technology-dependent starting from simple everyday activities like emails, mobile phones to complex applications such as manufacturing industries and aviation control. Failure to meet up these ever increasing demands even for a short while would lead to huge losses and hindrance to
\end{abstract}

This is an open access article under the CC BY-NC-ND license (https://creativecommons.org/licenses/by-nc-sa/4.0) 
development. To handle the enormous consumer needs of the world today, huge high speed data centres have been established such as Amazon, Yahoo, Google and eBay ${ }^{6}$ which led to the emergence of cloud computing as technology evolved making processing and storing faster, cheaper and more powerful.

Cloud computing has been defined by various authors in different perspectives. ${ }^{6}$ considered cloud computing in relation to its utility to end-user and defined it as "a market-oriented distributed computing system consisting of a collection of interconnected and virtualized computers that are dynamically provisioned and presented as one or more unified computing resource(s) based on service-level agreements established through negotiation between the service provider and consumers", whereas National Institute of Standards and Technology ${ }^{27}$ defined cloud computing as "a model for enabling convenient, on- demand ${ }^{6}$ network access to a shared pool of configurable computing resources (e.g. networks, servers, storage, applications, and services) that can be rapidly provisioned and released with minimal management effort or service provider interaction". Cloud service centres are virtualised and distributed geographically providing services and applications at high processing speeds thereby requiring a huge amount of energy for its various operations. With the increased amount of cloud services consumed, various studies have been conducted which revealed alarming results in relation to its effects on the environment. ${ }^{23}$ states that 170 million metric tons of carbon can be produced by one data centre per year. Rivoire et al., ${ }^{21}$ carried out a study where it was reported that the information and communication technology sector produces an alarming amount of carbon (IV) oxide (CO2) that is equivalent to the amount released by the aviation industry and also amounts to $2 \%$ of world's emission. ${ }^{12}$ mentioned that the power consumed by the datacentre of Microsoft alone in Quincy, USA, is capable of supplying electricity to 40,000 houses. In order to handle the consumer needs, data centre electricity consumption increased by $56 \%$ from 2005 to $2010^{15.25}$, states that carbon footprint will increase by $20 \%$ by the year 2020 which is mainly because of the cloud computing architectures used by the data centres. This gave rise to huge concerns in the information technology world requiring immediate researches and studies to be carried out in order to significantly reduce greenhouse gas emissions making the world environmental friendly and hence, the term green computing or green IT.

Green computing is a phenomenon that is gaining significance and importance as technology dependency increases. It encompasses practices in the IT field that are eco-friendly so as to have minimum adverse effects in terms of e-waste generation, carbon footprint emissions, energy consumption and recycling on the environment. It is being implemented in different sectors of the IT field as it reduces energy consumption thereby reducing cost and also the harmful effects on the environment. Green computing can be applied in every field of IT application and can even have immediate effects such as turning off computers, storage and network devices when idle, use of flat screen computers to reduce energy consumption, proper disposal and recycling of gadgets to avoid the release of harmful elements into the environment. Regular practice and implementation of these healthy habits can lead to higher and better sustainability of the environment.

Other efforts have been made to make cloud computing green as the rate of energy consumption, carbon emission, e-waste disposal increased by implementing resource virtualisation and resource consolidation $^{7}$ in achieving a better energy efficiency in cloud environments. According to a research carried out by Accenture Microsoft Report (2010), carbon emissions from business applications performed on cloud can be significantly reduced depending on the size of the organisation. It was seen that small organisations reduced carbon footprint the most, amounting to a $90 \%$ reduction, mid-size organisations reduce emissions in the range of $60-90 \%$, while large organisations reduction range is $30-60 \%$. On the other hand however, Greenpeace International (2010) argues that carbon footprint emissions from cloud computing may only have more devastating effects as service providers are more concerned in the economic effect of reducing power consumption rather than harmful greenhouse gas (GHG) emissions.

\section{Achieving Green Cloud Computing via Energy} Efficiency : 
Virtualisation is the ability to run multiple services and applications from a single server. This helps in reducing physical server footprint needed, less infrastructure to execute the workloads and also less energy consumption as a result of less number of physical equipment. Virtualisation takes into consideration consolidation and virtual machine (VM) migration reducing VM overhead and improving energy efficiency. Consolidation controls the trade-off of performance, resource utilisation and energy expended $^{24}$ while VM migration provides flexible resource allocation and crisis management ${ }^{5}$. AbdelSalam et al., ${ }^{1}$ proposed an optimisation model for energy consumption of cloud environments. However, the problem of resource underutilization and over-provisioning of servers was addressed by Ranganathan et al., ${ }^{20}$.

Research has also been carried out on storage devices and systems to improve energy efficiency. Gurumurthi, Sankar and Stan, ${ }^{9}$ worked on high storage drives to improve disk bandwidth without increasing energy dissipation. Kaushik et al., ${ }^{13}$ postulated such a system by classifying storage serves into logical hot and cold zones and then switching to inactive states to conserve energy. Verma et al., ${ }^{26}$ presented an optimization for storage virtualization based on input output workload intensity.

Due to the large quantity of energy consumed in cloud computing data centres, a huge amount of heat is dissipated which in turn affects the global climate. To address this issue, several researches have been carried out. Moore, Chase and Ranganathan, ${ }^{20}$ researched on automatic reconfiguration of thermal load management system to improve cooling efficiency and power consumption. Bash and Forman, ${ }^{4}$ designed a mathematical model to improve the cooling of data centres while Ramos and Bianchini, ${ }^{19}$ provided a software prediction for thermal management of data centres based on load redistribution.

Increased energy consumption and capital required to run data centres have led to cloud service providers to opt for renewable energy generation using water, wind or sun. Based on these criteria, the location of data centres are decided giving priority to weather, availability of renewable energy sources. Cooling data centre equipment consume a high percentage of energy and to overcome this, air and water are used to achieve both the cooling effect and reduced carbon emission.

Another platform for IoT is fog computing, introduced in 2010 to solve the technical complexities of cloud computing, however, it is an add-on of cloud computing and cannot exists as a standalone approach $^{3}$. Fog computing transfers the core functions of cloud computing towards the edge of the network as billions of devices are internet-connected and some of these devices located at the edge of the network require support for low latency, real-time and location aware services which cannot be provided efficiently by the traditional architecture of cloud computing ${ }^{17}$. Jalali et al.," described it as "a platform for local computing, distribution and storage in end-user devices rather than centralized data centres".

Misra and Sarkar, ${ }^{17}$ established that fog and cloud computing supplement each other and is an efficient green computing platform when combined as results showed that the mean energy consumption in fog computing was $40.48 \%$ less than the traditional cloud architecture for a scenario where $25 \%$ of the IoT applications demanded real-time and low latency services.

Jalali et al., ${ }^{11}$ studied the energy consumption between running applications from very small servers (nano data centres) which are located in end-user premises and centralized data centres. The experiment showed that energy was saved for some applications which were run from the small servers as the content was closer to the end-user and this saved energy from the transport network, but more energy was consumed when the small servers were connected to an energyinefficient access network. However, some factors of the type of applications such as the amount of data pre-loading affected the energy consumption rate and energy was efficiently saved from applications running in the small servers which had low access data rate such as video surveillance.

$5 \mathrm{G}$ network will also benefit from the cloudfog architecture as a study carried out by Kitanov and Janevski, ${ }^{14}$ has shown that $5 \mathrm{G}$ network is more energy efficient than $3 \mathrm{G}$ or $4 \mathrm{G}$ when the number of smart device users increases or the distance of this user increases from a radio access network (RAN). 
Fog computing has also been used for processing and analysing electrocardiogram (ECG) signals sent by patients via a heart monitoring application. It proved to be more energy efficient by saving up to $68 \%$ energy than processing the ECG signals at the cloud as studied by Isa et al. ${ }^{10}$. This is because the energy consumed by the transport equipment to the clouds reduced as the processing servers were situated closer to the end-user.

Li et al. ${ }^{16}$ reported that $80 \%$ of energy used in the world today is still produced from fossil fuels and in order to make edge computing green, not only energy efficient techniques should be utilised or improved, but also the primary source of power should be renewable energy sources while fossil fuels could be used as the secondary source of power. When using renewable energy, energy allocation is a crucial issue, and this requires that the usage profile of the edge computing systems be drawn. The study also reported that unpredictability and cost are the two major factors hindering the usage of renewable energy and with the help of microgrid, renewable energy sources in a particular area can be combined to distribute power to local users and the demands can be satisfied in a convenient way while reducing the carbon footprint. To reduce carbon emission, a prototype system using an edge computing and microgrid to integrate highly volatile renewable energy sources in an area, was built and it was observed that green power was supplied to the edge computing system 94.8 percent of the experimental time.

Sarkar, Chatterjee and Misra, ${ }^{22}$ studied the amount of $\mathrm{CO}_{2}$ gas emitted in terms of three processes which were packet forwarding, storage and computation in both cloud computing and fog computing platforms. The results indicated that when non-renewable energy sources such as diesel, coal and natural gas were used to supply energy to each of the data centre architectures, there was a significant decrease in $\mathrm{CO}_{2}$ emission in fog computing than the traditional cloud computing. Renewable energy sources such as geothermal, hydroelectric and offshore wind were also used to determine the $\mathrm{CO}_{2}$ emission in the three processes mentioned earlier and the results indicated that fog computing was greener compared to its counterpart.

\section{Challenges of Efficient Green Cloud Computing :}

Technological advances in virtually all aspects of life have led to a high dependency on computerisation and automation. This has led to the concept of cloud computing to handle the ever increasing demand of services and applications across the globe via high speed processing networks. However, the downside of this is the increased emission of harmful greenhouse gases into the atmosphere, improper disposal and recycling of electronic products, high energy consumption and heat generation. To reduce the alarming effects of technological advances on the environment, scientists and researchers now focus on green computing of clouds.

Several studies have been conducted. However, there are still some shortcomings which can be addressed such as study of heterogeneous work loads of VMs, for example, implementing a high processing activity (utilises processing power) and a high storage activity (storage devices). This will lead to increased resource utilisation and also avoid wastage of servers since one server may do the job of multiple servers thereby reducing heat generation and energy consumption. Cooling methods are provided to address the heat dissipated from hardware. However, studies still lack on the generation of heat by software. Studies should be carried out on compatibility of various softwares and services running concurrently on servers to determine which software-service group generates least heat with high energy and performance efficiency. Fog computing could be used as an alternative to push content closer to users. However, there is need to identify and understand the applications which should be run from the edge of the network to save energy consumption. Apart from improving the techniques, renewable energy should be used as the primary source to supply power, but using these sources are very expensive and unpredictable. It has been seen that it is feasible to use a microgrid to combine the renewable energy in an area to supply green energy to edge computing systems, but an energy management scheme should be developed when using it in real-life scenarios.

Numerous advancements have been made to address the effects of cloud computing on the global 
climate. However, economic advantage is considered more than the environmental advantage, that is, service providers ultimately reduce energy consumption by utilising cheaper energy sources which may not be necessarily healthier. It is, however, recommended to implement strong policies to back green computing for a healthy future. Also, all data centres should increase the amount of renewable energy generation and move towards a low dependency on unhealthy perishable forms of energy like coal.

\section{Conclusion and Future Direction}

Carbon emission by technological advances and dependence on computerisation are major issues. The focal purpose of this paper is to summarize the causes of e-waste production by cloud computing, the importance of mitigating the GHGs produced by the clouds, ways to practice green cloud computing, and also areas where development is required to assist in achieving a green and healthier environment.

It has been observed from previous studies that fog computing architecture proved to be greener than using only cloud computing architectures. Therefore, as the world gradually evolves into automating every aspect and sector of life, the future direction of this study would be to integrate fog computing with artificial intelligence techniques for smart and automated energy efficiency determination and regulation.

\section{References}

1. AbdelSalam, H., Maly, K., Mukkamala, R., Zubair, M. and Kaminsky, D., 'Towards Energy Efficient Change Management in a Cloud Computing Environment', in Sadre, R. and Pras, A. (eds.) Scalability of Networks and Services, Third International Conference on Autonomous Infrastructure, Management and Security, AIMS 2009. Enschede, The Netherlands: Springer Berlin Heidelberg, pp. 161-166(2009).

2. Accenture Microsoft Report, Cloud computing and Sustainability: The Environmental Benefits of Moving to the Cloud. Available at: http:// www.wspenvironmental.com/media/docs/
newsroom/Cloud_computing_and_ Sustainability_Whitepaper_-_Nov_2010.pdf (Accessed: 10 December 2012) (2010).

3. Aljumah, A. and Ahanger, T.A., 'Fog computing and security issues: A review', 2018 7th International Conference on Computers Communications and Control (ICCCC). Oradea, Romania: IEEE, pp. 237-239 (2018).

4. Bash, C. and Forman, G., 'Cool Job Allocation: Measuring the Power Savings of Placing Jobs at Cooling-efficient Locations in the Data Center', 2007 USENIX Annual Technical Conference on Proceedings of the USENIX Annual Technical Conference. Santa Clara, CA: USENIX Association, p. 29:1-29:6 (2007).

5. Beloglazov, A., Buyya, R., Lee, Y.C. and Zomaya, A., 'A Taxonomy and Survey of Energy-Efficient Data Centers and Cloud Computing Systems', Advances in Computers, 82, pp. 47-111 (2010).

6. Buyya, R., Yeo, C.S. and Venugopal, S., 'MarketOriented Cloud Computing: Vision, Hype, and Reality for Delivering IT Services as Computing Utilities', 2008 10th IEEE International Conference on High Performance Computing and Communications. Dalian, China: IEEE, pp. 5-13 (2008).

7. Garg, S.K. and Buyya, R., 'Green cloud computing and environmental sustainability', Harnessing Green IT: Principles and Practices, , pp. 315340 (2012).

8. Greenpeace International, Make IT Green. Available at: http://www.greenpeace.org/ international/en/publications/reports/make-itgreen-cloudcomputing (Accessed: 10 December 2012) (2010).

9. Gurumurthi, S., Sankar, S. and Stan, M.R., 'Using Intradisk Parallelism to Build Energy-Efficient Storage Systems', IEEE Micro, 29(1), pp. 50-61 (2009).

10. Isa, I.S.M., Musa, M.O.I., El-Gorashi, T.E.H., Lawey, A.Q. and Elmirghani, J.M.H., 'Energy Efficiency of Fog Computing Health Monitoring Applications', 201820th International Conference on Transparent Optical Networks (ICTON). IEEE, Vol. 2018-July, pp. 1-5 (2018).

11. Jalali, F., Hinton, K., Ayre, R., Alpcan, T. and Tucker, R.S., 'Fog Computing May Help to Save 
Energy in Cloud Computing', IEEE Journal on Selected Areas in Communications, 34(5), pp. 1728-1739(2016).

12. Katz, R.H., 'Tech Titans Building Boom', IEEE Spectrum, 46(2), pp. 40-54 (2009).

13. Kaushik, R.T., Cherkasova, L., Campbell, R. and Nahrstedt, K., 'Lightning: Self-adaptive, energyconserving, multi-zoned, commodity green cloud storage system', Proceedings of the 19th ACM International Symposium on High Performance Distributed Computing - HPDC '10. New York, New York, USA: ACM Press, p. 332 (2010).

14. Kitanov, S. and Janevski, T., 'Energy efficiency of Fog Computing and Networking services in 5G networks', IEEE EUROCON 2017 -17th International Conference on Smart Technologies. IEEE, pp. 491-494(2017).

15. Koomey, J.G., 'Growth in Data Center Electricity Use 2005 to 2010', A report by Analytical Press, completed at the request of The New York Times, 9, p. 161 (2011).

16. Li, W., Yang, T., Delicato, F.C., Pires, P.F., Tari, Z., Khan, S.U. and Zomaya, A.Y., 'On Enabling Sustainable Edge Computing with Renewable Energy Resources', IEEE Communications Magazine, 56(5), pp. 94-101 (2018).

17. Misra, S. and Sarkar, S., 'Theoretical modelling of fog computing: a green computing paradigm to support IoT applications', IET Networks, 5(2), pp. 23-29 (2016).

18. Moore, J., Chase, J.S. and Ranganathan, P., 'Weatherman: Automated, Online and Predictive Thermal Mapping and Management for Data Centers', 2006 IEEE International Conference on Autonomic Computing. Dublin, Ireland: IEEE, pp. 155-164 (2006).

19. Ramos, L. and Bianchini, R., 'C-Oracle: Predictive thermal management for data centers', 2008 IEEE 14th International Symposium on High Performance Computer Architecture. Salt Lake City, UT, USA: IEEE, pp. 111-122 (2008).

20. Ranganathan, P., Leech, P., Irwin, D. and Chase, J., 'Ensemble-level Power Management for Dense Blade Servers', 33rd International Symposium on Computer Architecture (ISCA'06). Boston, MA, USA: IEEE, pp. 66-77 (2006).

21. Rivoire, S., Shah, M.A., Ranganathan, P. and Kozyrakis, C., 'JouleSort: A balanced energyefficiency benchmark', Proceedings of the 2007 ACM SIGMOD international conference on Management of data - SIGMOD '07. New York, New York, USA: ACM Press, p. 365 (2007).

22. Sarkar, S., Chatterjee, S. and Misra, S., 'Assessment of the Suitability of Fog Computing in the Context of Internet of Things', IEEE Transactions on Cloud Computing, 6(1), pp. 46-59 (2018).

23. Smith, S.F., 'Is Scheduling a Solved Problem?', in Kendall, G., Burke, E.K., Petrovic, S., Gendreau, M. (ed.) Multidisciplinary Scheduling: Theory and Applications. UK: Springer, pp. 3-17 (2005).

24. Srikantaiah, S., Kansal, A. and Zhao, F., 'EnergyAware Consolidation for Cloud Computing', Cluster Computing - CLUSTER, 12, pp. 10-10 (2008).

25. Thakur, S. and Chaurasia, A., 'Towards Green Cloud Computing: Impact of carbon footprint on environment', 2016 6th International Conference - Cloud System and Big Data Engineering (Confluence). IEEE, pp. 209-213 (2016).

26. Verma, A., Koller, R., Useche, L. and Rangaswami, R., 'SRCMap: Energy Proportional Storage Using Dynamic Consolidation', 8th USENIX Conference on File and Storage Technologies. San Jose, CA, USA, pp. 267-280 (2010).

27. Mell, P.M. and Grance, T., The NIST definition of cloud computing. Gaithersburg, MD. (2011). 